\title{
The Placement of Post-pyloric Feeding Tubes Using DRX-Revolution Mobile X-Ray System in an ICU. A Case Series
}

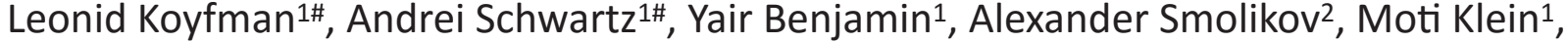 \\ Evgeni Brotfain ${ }^{*}$ \\ 1 Department of Anesthesiology and Critical Care, Soroka Medical Center, Ben-Gurion University of the Negev, Beer \\ Sheva, Israel \\ 2 Department of Radiology, Soroka Medical Center, Ben-Gurion University of the Negev, Beer Sheva, Israel
}

\begin{abstract}
Enteral nutrition is crucial for ensuring that critically ill patients have a proper intake of food, water, and medicine. Methods to ensure this requirement should be initiated as early as possible. The use of PPF has several advantages compared to the use of a nasogastric feeding tube. In the present paper, the cases of three critically ill patients with a nonfunctional gastrointestinal system on admission to ICU, are detailed. Enteral feeding through a nasogastric tube by prokinetic agent therapy had been unsuccessful. The bedside placement of a post-pyloric feeding tube by the DRX-Revolution X-ray system is described.
\end{abstract}

Keywords: post-pyloric feeding tube (PPF), DRX-Revolution X-ray system, enteral nutrition

Received: 05 April 2016 / Accepted: 20 May 2016

\section{INTRODUCTION}

Enteral nutrition is crucial for ensuring proper intake of food, water, and medicine by critically ill patients and should be initiated as early as possible [1]. Both nasogastric and the post-pyloric feeding tubes (PPF) are frequently used in intensive care units (ICUs) throughout the world. The use of PPFis associated with several advantages compared to the use of a nasogastric feeding tube, such as lower rates of ventilator-associated pneumonia due to the reduced risk of aspiration pneumonia, shorter stay in an intensive care unit (ICU) [2] and better caloric intake [2-4]. The placement of a PPF can be accomplished using radiologic, electromagnetic, or endoscopic techniques, or without reliance on other means such as blind placement [4-5].

The DRX-Revolution Mobile X-ray system is a portable, bedside X-ray imaging system that features higher resolution and improved radiation doses per image. In the present report, three clinical cases of successful PPF placement using the DRX-Revolution X-ray system are described.

\section{CASE 1}

Following a motor vehicle accident, a 61-year-old male was transferred to the ICU from the emergency room, with multiple trauma injuries. He had blunt thoracic trauma with multiple rib fractures, hemopneumothorax, spleen laceration Grade 2, as well as a D10-D12 vertebra, left acetabulum, and bilateral malleolus fractures. He was admitted to the ICU in a hemodynamically unstable condition having a systolic blood pressure $<70 \mathrm{mmHg}$, and tachycardia $>130$ beats/minute. He was breathing spontaneously and was fully conscious. During the following forty eight hours, due to deteriorated respiratory function, the patient was sedated and ventilated. Upon admission to the ICU, a nasogastric tube was inserted, and enteral feeding was started. Two days later, high gastric residual volumes $(500-800 \mathrm{ml}$

\footnotetext{
\# Both authors contributed equally.

Correspondence to: Evgeni Brotfain, Ben-Gurion University of the Negev, PO Box 653, Beer Sheva, 8499000, Izrael. Tel:+972546218106. E-mail: bem1975@gmail.com Leonid Koyfman: Soroka Medical Center, Yitzhak Rager Boulevard 151, Beer Sheva, Izrael. Tel:++972 8-640-0111. E-mail: leonidko@gmail.com

Andrei Schwartz: Soroka Medical Center, Yitzhak Rager Boulevard 151, Beer Sheva, Izrael. Tel:++972 8-640-0111. E-mail: schwartz72@hotmail.com

Yair Benjamin: Ben-Gurion University of the Negev, PO Box 653, Beer Sheva, 8499000, Izrael. Tel:+972546218106

Alexander Smolikov: Soroka Medical Center, Yitzhak Rager Boulevard 151, Beer Sheva, Izrael. Tel:++972 8-640-0111

Moti Klein: Ben-Gurion University of the Negev, PO Box 653, Beer Sheva, 8499000, Izrael Tel:+972546218106
} 
every 8 hours) and vomiting were observed. CT abdomen imaging demonstrated distention of the stomach and the small intestine. There were no signs of bowel obstruction or active bleeding from the spleen laceration. Initial management of gastroparesis and paralytic ileus with prokinetic agents, metoclopramide, and erythromycin was unsuccessful.

The following day, a PPF tube was placed using the DRX-Revolution Mobile X-ray system, each image of being about $0.05 \mathrm{mSv}$ (Figures 1a-1c).

The PPF was advanced from the nasopharynx and inserted into the stomach. The first X-ray image was taken to confirm the PPF position in the stomach, (Figure 1a) then advanced further, and another two X-ray images were acquired, to verify its placement in the post-pyloric area, specifically, in the second portion of the duodenum (Figures 1a-1c).

There were no complications either during or after the procedure. The PPF placement took approximately twenty minutes. Adequate nutrition supply and caloric intake were achieved during the ICU stay. Over the next ten days, the patient underwent surgical fixation of the bilateral malleolus fractures. The patient was weaned from mechanical ventilation, extubated, and finally discharged from the ICU two weeks after admission.

\section{CASE 2}

A 53-year-old male initially presented at the General ICU with $25 \%$ burns on the chest, upper extremities,

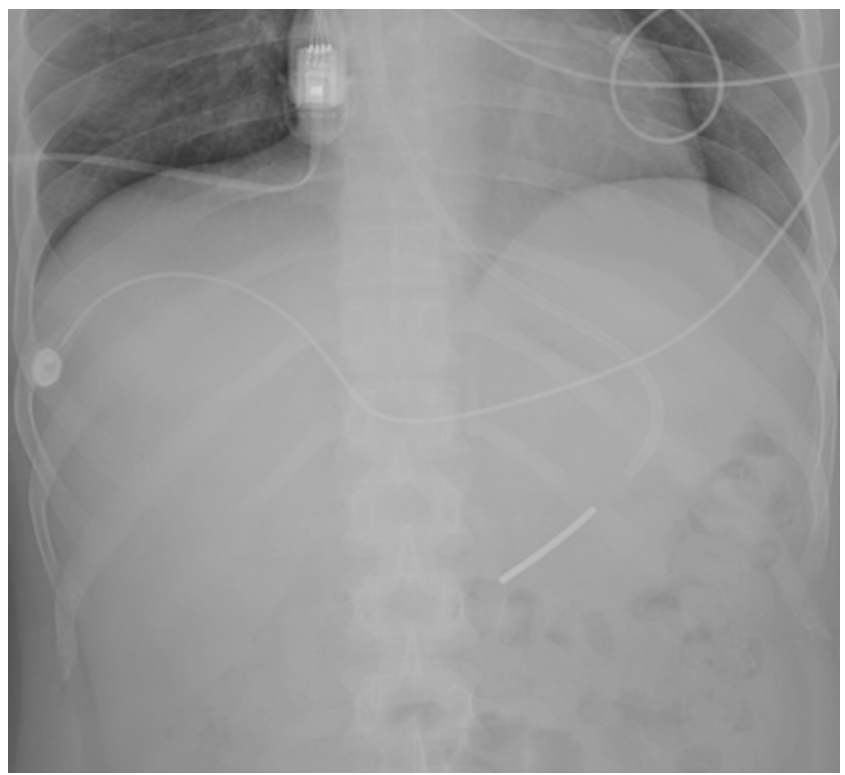

Fig. 1a. PPF position in the stomach using DRX-Revolution Mobile X-ray system. and back. During the first seventy-four hours, surgical debridement of the burn was performed several times. At the same time, the patient became septic and developed multiple organ failures, and showed severe respiratory, renal and cardiovascular dysfunction. A physical examination revealed a distended abdomen and the absence of bowel sounds. An abdomen X-ray confirmed the diagnosis of paralytic ileus. The patient was treated with prokinetic agents and electrolyte supply. The nasogastric feeding tube was replaced with a PPF tube, using the DRX-Revolution Mobile X-ray system. The PPF position was verified by three images obtained via the DRX-Revolution Mobile X-ray system, using a total dose of $0.15 \mathrm{mSv}$. No complications were observed. Adequate nutrition supply and caloric intake were achieved over the following 48 hours.

\section{- CASE 3}

A 36-year-old male was transferred from the operating room to the ICU after an urgent exploratory laparotomy was performed related to intrabdominal sepsis. A week beforehand, the patient had undergone bariatric surgery, by a sleeve gastrectomy procedure. The patient was diagnosed as being septic, having a WBC 21000 / ul, neutrophils- $94 \%$, with multiple organ failures, sinus tachycardia 126 beats $\backslash$ minute, systemic blood pressure $79 \backslash 41 \mathrm{mmHg}$, lactate $>3 \mathrm{mmol} / \mathrm{L}$, ph-7.2, creatinine 140 $\mu \mathrm{mol} / \mathrm{l}$. During laparotomy, a leak from the the lower esophageal region was identified. The lower esophageal region was explored and re-sutured. Parenteral nutri-

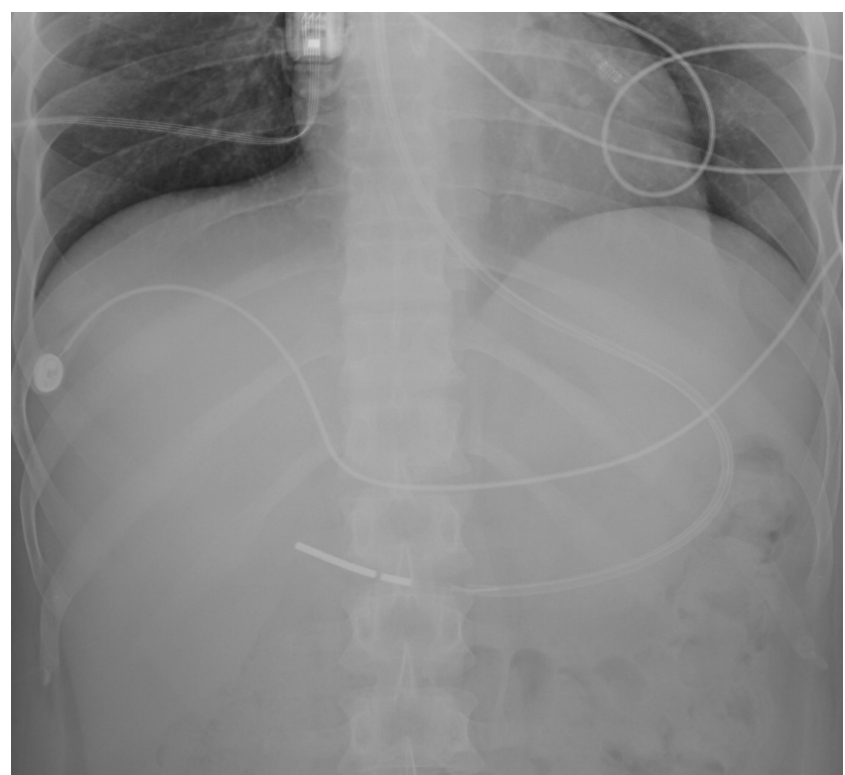

Fig. 1b. PPF position in pylorus (using DRX-Revolution Mobile X-ray system). 


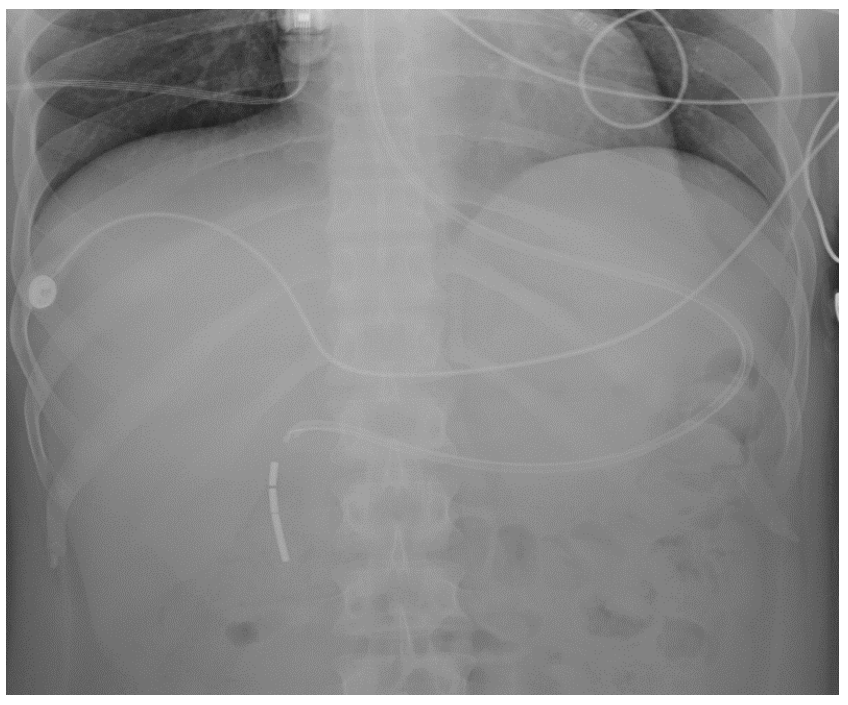

Fig. 1c. PPF in post-pyloric position (using DRX-Revolution Mobile X-ray system).

tion was initiated immediately on the day of admission to the ICU and maintained over the next three weeks. Finally, given improvements in the patient's condition and in conjunction with a surgical consultant, it was decided to start enteral feeding. PPF placement was performed at the bedside, using the DRX-Revolution Mobile X-ray system imaging, using three images, each $0.06 \mathrm{mSv}$ each. No complications occurred during or after the procedure. Enteral feeding through the PPF continued successfully and uneventfully until the patient was discharged from the ICU to the surgical ward.

\section{DISCUSSION}

A constancy of enteral nutrition during hospitalization in the ICU and especially in the first 36-48 hours is crucial for the critically ill patient, as it makes it possible to maintain mucosal integrity and mucosal immunity, with a reduction in infectious complications $[6,7]$.

Critically ill patients commonly develop intolerance to gastric feeding because of the decrease in gastric motility following injury, critical illness, or the administration of drugs such as opiates, benzodiazepines, muscle relaxants, or catecholamines [8]. In such cases, PPF is very useful. PPF tubes can supply enteral nutrition, water, and the administration of medication. Moreover, previously published data has demonstrated that PPF feeding reduced the risk of gastroesophageal reflux and of aspiration pneumonia [8,9]. In contrast, Marik and Zaloga [10] did not find that the route of feeding (gastric or post-pyloric) had any influence on mortality, days in the ICU, caloric intake, or incidence of pneumonia.

For patients with certain critical gastrointestinal illnesses, such as acute pancreatitis, gastroparesis, gastric feeding intolerance, or severe gastroesophageal reflux, as well as in cases of previous gastric surgery or major abdominal surgery, there might be a need to use PPF tubes, to begin with, upon admission to the ICU [6].

PPF tubes are inexpensive, the placement procedure can be conducted at the bedside, and their removal is easily managed [6]. However, some risks and complications can result from PPF tube placement $[6,8,9]$. The major risk is inadvertent misplacement, especially into the lung. Also, PPF tubes may become dislocated or obstructed before the patient can resume oral intake and before a more permanent and regular feeding method is established [11].

There are various techniques for placing and positioning PPF tubes. A blind insertion of PPF is the most popular and may be performed by ICU physicians, trained registered dietitians, or ICU registered nurses [9]. Every blind PPF tube placement needs to be confirmed via abdominal X-ray [9]. Gatt and MacFie reported an $80 \%$ success rate of PPF tube placement using the blind bedside technique [12]. PPF tube placement can be facilitated by the use of several different technologies, such as endoscopic placement [8], magnetic-assisted placement, electrocardiographic guidance, prokinetic agents such as metoclopramide and erythromycin, usage of special feeding tubes, and air insufflations [8]. However, some of these are high-cost techniques, which also require special practical skills [8] Endoscopic placement of PPF tubes was demonstrated to have a high rate of success, up to $90 \%$ [13].

Thurley et al. [14] described the placement of 200 PPF tubes in surgical and oncological patients using fluoroscopic insertion. All procedures were performed in the radiology department [14]. 3.5\% of the immediate and $20 \%$ of late complications observed were related to vomiting.

The DRX-Revolution X-ray system features a unique tube-and-grid alignment system that delivers superb $\mathrm{X}$-ray quality and encourages grid use [15] It also has better image processing, allowing for a quick and accurate diagnosis [15]. It is also a mobile system, with the ability to move in horizontal, vertical, tilted, angulated, and rotated aspects [15]. It is said to reduce radiation dose by $30-50 \%$ [15]. 
In the three reported cases, patients received a dose of 0.05-0.06 $\mathrm{mSv}$ per image. Three images were needed in each case to ensure appropriate PPF positioning. This dose was considered to be $50 \%$ less than the dose of a routine chest X-ray examination.

In a study by Thurley et al., [14] all procedures were conducted in a general screening room using a Philips Diagnostic 56 Radiography /Fluoroscopy system (Philips V3000DSA unit). Moreover, there was no specification of the total dose of radiation or the number of images needed to position the PPF. In the current cases, all of the images were acquired at the bedside, thus underscoring an additional clinical advantage of the DRX-Revolution X-ray system, namely, averting the need to transport the patients out of the ICU and instead allowing them to remain under individual and vigilant nursing care.

\section{CONCLUSION}

The reported cases suggest that the DRX-Revolution $\mathrm{X}$-ray system provides safe PPF placement and is a promising and beneficial technique for use in bedside procedures in an ICU.

\section{CONFLICT OF INTEREST}

None declared

\section{REFERENCES}

1. Swinton F, Donovan $\mathrm{K}$ and White $\mathrm{N}$. Insertion of post-pyloric feeding tubes in the ICU is not as difficult as we thought. Journal of Intensive Care Society. 2009;10:273-4.

2. Kearns J, Chin D, Mueller L et al. The incidence of ventilatorassociated pneumonia and success in nutrient delivery with gastric versus small intestinal feeding: a randomized clinical trial. Crit Care Med, 2000;28:1742-6.

3. Montejo JC, Grau T, Acosta J et al. Multicenter, prospective, randomized, single-blind study comparing the efficacy and gastrointestinal complications of early jejunal feeding with early gastric feeding in critically ill patients. Crit Care Med. 2002;30:796-800.

4. Stone SJ, Pickett JD, Jesurum JT. Bedside placement of postpyloric feeding tubes. AACN Clin Issues. 2000;11:517-30.

5. Lenart S, Polissar NL. Comparison of 2 methods for postpyloric placement of enteral feeding tubes. Am J Crit Care. 2003;12:35760.

6. Aguilar-Nascimentoa JE, Kudsk KA. Use of small-bore feeding tubes: successes and failures. Current Opinion in Clinical Nutrition and Metabolic Care. 2007;10:291-6.

7. Peter JV, Moran JL, Phillips-Hughes J. A metaanalysis of treatment outcomes of early enteral versus early parenteral nutrition in hospitalized patients. Crit Care Med. 2005;33:21320.

8. Tiancha H, Jiyong J, Min Y. How to Promote Bedside Placement of the Postpyloric Feeding Tube: A Network Meta-Analysis of Randomized Controlled Trials. JPEN J Parenter Enteral Nutr. 2015;39:521-30.

9. Rollins CM. Blind Bedside Placement of Postpyloric Feeding Tubes by Registered Dietitians: Success Rates, Outcomes, and Cost Effectiveness. Nutr Clin Pract. 2013;28:506-9.

10. Marik PE, Zaloga GP. Gastric versus postpyloric feeding: a systematic review. Crit Care. 2003;7:R46-R51.

11. Baskin WN. Acute complications associated with bedside placement of feeding tubes. Nutr Pract Clin. 2006;21:40-55.

12. Gatt M, MacFie J. Bedside postpyloric feeding tube placement: A pilot series to validate this novel technique. Crit Care Med. 2009; 37:523-7.

13. Fang JC, Hilden K, Holubkov R, DiSario JA. Transnasal endoscopy vs. fluoroscopy for the placement of nasoenteric feeding tubes in critically ill patients. Gastrointest Endosc. 2005;62:661-6.

14. Thurley PD, Hopper MA, Jobling JC and Teahon. Fluoroscopic insertion of post pyloric feeding tubes: success rates and complications. Clin Radiology. 2008;63:543-8.

15. http://www.carestream.com/drx-revolution.html 